\title{
Evaluating the role of neoadjuvant chemotherapy in bladder cancer patients with occult lymph node metastases
}

\author{
Marco Moschini ${ }^{1,2}$, Stefania Zamboni ${ }^{1}$, Agostino Mattei ${ }^{1}$, Alberto Martini ${ }^{2}$, Emanuele Zaffuto ${ }^{2}$, Alberto \\ Briganti ${ }^{2}$, Andrea Gallina ${ }^{2}$, Francesco Montorsi ${ }^{2}$ \\ ${ }^{1}$ Klinik für Urologie, Luzerner Kantonsspital, Lucerne, Switzerland; ${ }^{2}$ Unit of Urology/Division of Oncology, IRCCS Ospedale San Raffaele, URI, \\ Milan, Italy \\ Correspondence to: Marco Moschini, MD, PhD. Klinik für Urologie, Luzerner Kantonsspital, CH-6000, Luzern 16, Switzerland. \\ Email: marco.moschini87@gmail.com. \\ Provenance: This is a Guest Editorial commissioned by Section Editor Xiao Li (Department of Urology, Jiangsu Cancer Hospital \& Jiangsu Institute \\ of Cancer Research \& Nanjing Medical University Affiliated Cancer Hospital, Nanjing, China). \\ Comment on: Cha EK, Sfakianos JP, Sukhu R, et al. Poor prognosis of bladder cancer patients with occult lymph node metastases treated with \\ neoadjuvant chemotherapy. BJU Int 2018. [Epub ahead of print].
}

Submitted Jul 01, 2018. Accepted for publication Jul 03, 2018.

doi: $10.21037 /$ tau. 2018.07 .03

View this article at: http://dx.doi.org/10.21037/tau.2018.07.03

Bladder cancer (BCa) is the second most common genitourinary malignancy with 81,190 estimated new diagnosis in the 2018 in the United States only (1). Radical cystectomy (RC) with bilateral pelvic node dissection (PLND) represents the gold standard for very recurrent high risk non-muscle invasive tumors and for muscle invasive $\mathrm{BCa}$ (2). However, despite surgery, up to $50 \%$ of patients experience disease recurrence and succumb from their disease $(3,4)$. To improve these poor survival expectations, neoadjuvant chemotherapy (NAC) has been proved by level one evidences $(5,6)$ to improve overall survival of $8 \%$ at 5 years in cT2-T4a N0 M0 BCa patients. However, the non-negligible toxicity rate [grade 3 and 4 reported in $35 \%$ and $37 \%$ of patients, respectively (5)] and other elements such as patient characteristics and surgeons' preferences limit the widespread of NAC, that, despite an increasing trends with a peak of $20.9 \%$ in 2010 for patients potentially eligible (7), remains underused. To increase this unsatisfactory appliance rate, several authors tried to build preoperative models to help clinicians to identify the best responders to NAC in an effort to reduce unnecessary complications and to maximize the survival benefit (8). Specifically, patients diagnosed with locally advanced disease (clinical stage $\geq \mathrm{T} 3$ ) are those who had the greater survival advantage after NAC treatment and are those who might benefit more from NAC $(9,10)$. This effect might be related to the higher tendency of locally advance BCa to metastasize with evident or occult lymph node metastases. On the other hand, the poor performance characteristics of cross sectional imaging (11) represents a huge limitation in defining the presence of clinical node metastases prior to $\mathrm{RC}$ and at the time only an extended and well executed PLND can adequately assess the presence of node metastases (12).

In this regard, Cha et al. (13) analyzed an unmet question, trying to describe the survival outcomes of patients with occult lymph node metastases treated with NAC by comparing them to patients treated without NAC and with subsequent adjuvant chemotherapy for $\mathrm{pN} 1$ disease. The study population was represented by 198 patients treated with RC and PLND between 2000 and 2010 due to cN0M0 BCa who were found with node metastases at the pathological evaluation after surgery. The authors compared the effect of NAC $(\mathrm{N}=32)$ versus the effect of adjuvant chemotherapy $(\mathrm{N}=49)$ in this subgroup of patients $(\mathrm{pN} 1)$. They observed that $\mathrm{pN} 1$ patients previously treated with NAC have poor prognosis, significantly worse compared to those who were treated with adjuvant chemotherapy only.

Several considerations can be extrapolated from these results. First, it has to be taken into account the selection bias introduced in this retrospective analysis. In fact, although the patients with node metastases treated with 
adjuvant chemotherapy represent a homogeneous group, those who experienced node metastases after NAC are a selection of the poor responders among the group of patients who were treated with NAC despite having occult lymph node metastases. Considering this aspect, it is not surprising to observe that the subgroup who represents the selection of patients with the worse characteristics (NAC patients) have worse survival when compared to a homogeneous population group (adjuvant chemotherapy patients). Second, the poor performance characteristics of the preoperative cross-sectional imaging in detecting node metastases might have influenced the selection of the two study groups. Third, the decision of performing NAC or adjuvant chemotherapy were not taken randomly but represented direct consequences of patients and tumor characteristics as well as surgeon decisions.

However, the authors of the manuscript have to be commended to present in their manuscript an understudied population, reporting the poor survival expectancies of cN0M0 ypN+ patients (three-year recurrence free survival rate: $26 \%$ ). These results highlight the need of new and more aggressive therapeutic strategies for these patients. Recently, Seisen et al. (14) reported data of patients treated with NAC and RC between 2006 and 2012 from the National Cancer Data Base, observing in $\mathrm{pT} 3 / \mathrm{T} 4$ and or $\mathrm{pN}+$ an improved survival outcome for those patients treated with adjuvant chemotherapy versus observation. As highlighted by Cha et al. (13), other therapeutic strategies are presently under evaluation, such as neoadjuvant pembrolizumab (15) or adjuvant nivolumab and atezolizumab that might increase survival especially in these subgroups of patients (affected by aggressive disease and poor cisplatin response).

Understanding the aggressiveness of the disease and the sensitivity to the cisplatin based chemotherapies represents an important issue of the $\mathrm{BCa}$ research and preliminary data showed the possibility to divide on the bases of molecular subtype the $\mathrm{BCa}$ to predict the response of cisplatin based chemotherapy $(16,17)$. While awaiting these parameters to be validated in clinical practice, preoperative factors who might help to identify patients who might not respond to cisplatin or affected to particularly aggressive BCa disease are urgently needed. In this context, the presence of histological variants [although with some limitations TUR specimen (18)] might help physicians to individuate those patients at major risk of harboring node metastases and those who might not respond adequately to cisplatin based chemotherapy.

\section{Acknowledgements}

None.

\section{Footnote}

Conflicts of Interest: The authors have no conflicts of interest to declare.

\section{References}

1. Siegel RL, Miller KD, Jemal A. Cancer statistics, 2018. CA Cancer J Clin 2018;68:7-30.

2. Alfred Witjes J, Lebret T, Compérat EM, et al. Updated 2016 EAU Guidelines on Muscle-invasive and Metastatic Bladder Cancer. Eur Urol 2017;71:462-75.

3. Stein JP, Lieskovsky G, Cote R, et al. Radical cystectomy in the treatment of invasive bladder cancer: long-term results in 1,054 patients. J Clin Oncol 2001;19:666-75.

4. Moschini M, Karnes RJ, Sharma V, et al. Patterns and prognostic significance of clinical recurrences after radical cystectomy for bladder cancer: A 20-year single center experience. Eur J Surg Oncol 2016;42:735-43.

5. Grossman HB, Natale RB, Tangen CM, et al. Neoadjuvant chemotherapy plus cystectomy compared with cystectomy alone for locally advanced bladder cancer. $\mathrm{N}$ Engl J Med 2003;349:859-66.

6. International Collaboration of Trialists; Medical Research Council Advanced Bladder Cancer Working Party (now the National Cancer Research Institute Bladder Cancer Clinical Studies Group); European Organisation for Research and Treatment of Cancer Genito-Urinary Tract Cancer Group; et al. International phase III trial assessing neoadjuvant cisplatin, methotrexate, and vinblastine chemotherapy for muscle-invasive bladder cancer: longterm results of the BA06 30894 trial. J Clin Oncol 2011;29:2171-7.

7. Zaid HB, Patel SG, Stimson CJ, et al. Trends in the utilization of neoadjuvant chemotherapy in muscleinvasive bladder cancer: results from the National Cancer Database. Urology 2014;83:75-80.

8. Moschini M, Soria F, Klatte T, et al. Validation of Preoperative Risk Grouping of the Selection of Patients Most Likely to Benefit From Neoadjuvant Chemotherapy Before Radical Cystectomy. Clin Genitourin Cancer 2017;15:e267-e273.

9. Winquist E, Kirchner TS, Segal R, et al. Neoadjuvant chemotherapy for transitional cell carcinoma of the 
bladder: a systematic review and meta-analysis. J Urol 2004;171:561-9.

10. Advanced Bladder Cancer Meta-analysis Collaboration. Neoadjuvant chemotherapy in invasive bladder cancer: a systematic review and meta-analysis. Lancet 2003;361:1927-34.

11. Moschini M, Morlacco A, Briganti A, et al. Clinical Lymphadenopathy in Urothelial Cancer: A Transatlantic Collaboration on Performance of Cross-sectional Imaging and Oncologic Outcomes in Patients Treated with Radical Cystectomy Without Neoadjuvant Chemotherapy. Eur Urol Focus 2016. [Epub ahead of print].

12. Moschini M, Karnes RJ, Gandaglia G, et al. Preoperative Favorable Characteristics in Bladder Cancer Patients Cannot Substitute the Necessity of Extended Lymphadenectomy During Radical Cystectomy: A Sensitivity Curve Analysis. Urology 2016;88:97-103.

13. Cha EK, Sfakianos JP, Sukhu R, et al. Poor prognosis of bladder cancer patients with occult lymph node metastases treated with neoadjuvant chemotherapy. BJU Int 2018. [Epub ahead of print]

14. Seisen T, Jamzadeh A, Leow JJ, et al. Adjuvant

Cite this article as: Moschini $M$, Zamboni S, Mattei A, Martini A, Zaffuto E, Briganti A, Gallina A, Montorsi F. Evaluating the role of neoadjuvant chemotherapy in bladder cancer patients with occult lymph node metastases. Transl Androl Urol 2018;7(4):742-744. doi: 10.21037/tau.2018.07.03
Chemotherapy vs Observation for Patients With Adverse Pathologic Features at Radical Cystectomy Previously Treated With Neoadjuvant Chemotherapy. JAMA Oncol 2018;4:225-9.

15. Necchi A, Briganti A, Raggi D, et al. Interim results from PURE-01: A phase 2, open-label study of neoadjuvant pembrolizumab (pembro) before radical cystectomy for muscle-invasive urothelial bladder carcinoma (MIUC). J Clin Oncol 2018;36:TPS533.

16. Seiler R, Ashab HAD, Erho N, et al. Impact of Molecular Subtypes in Muscle-invasive Bladder Cancer on Predicting Response and Survival after Neoadjuvant Chemotherapy. Eur Urol 2017;72:544-54.

17. Choi W, Ochoa A, McConkey DJ, et al. Genetic Alterations in the Molecular Subtypes of Bladder Cancer: Illustration in the Cancer Genome Atlas Dataset. Eur Urol 2017;72:354-65.

18. Moschini M, Shariat SF, Freschi M, et al. Is transurethral resection alone enough for the diagnosis of histological variants? A single-center study. Urol Oncol 2017;35:528. e1-528.e5. 